\title{
MONALEESA clinical program: a review of ribociclib use in different clinical settings
}

\author{
Denise A Yardley*,1 \\ ${ }^{1}$ Department of Medical Oncology, Sarah Cannon Research Institute \& Tennessee Oncology PLLC, Nashville, TN 37203, USA \\ *Author for correspondence: Tel.: +1 615320 5090; Fax: +1 615986 0029; dyardley@tnonc.com
}

\begin{abstract}
Ribociclib has received approval in the pre/peri- and postmenopausal disease settings on the basis of the MONALEESA trials. MONALEESA-2 demonstrated that ribociclib plus letrozole significantly improved progression-free survival compared with placebo plus letrozole as first-line therapy in postmenopausal patients with HR-positive, HER2-negative advanced breast cancer. Subsequently, ongoing trials reported significant progression-free survival improvements with ribociclib in combination with either fulvestrant in postmenopausal patients with advanced breast cancer who were either treatment naive or received $\leq 1$ line of prior endocrine therapy in the advanced disease setting (MONALEESA-3) or tamoxifen/nonsteroidal aromatase inhibitor with ovarian function suppression in pre/perimenopausal women (MONALEESA7). This review summarizes the MONALEESA clinical program. ClinicalTrials.gov identifiers: NCT01958021 (MONALEESA-2), NCT02422615 (MONALEESA-3), NCT02278120 (MONALEESA-7).
\end{abstract}

First draft submitted: 8 March 2019; Accepted for publication: 19 June 2019; Published online:

15 July 2019

Keywords: breast cancer $\bullet$ CDK4/6 inhibitor • MONALEESA-2 • MONALEESA-3 $\bullet$ MONALEESA-7 • postmenopausal - premenopausal • ribociclib

Ribociclib (Novartis Institutes for BioMedical Research in collaboration with Astex Pharmaceuticals) is an orally bioavailable, selective inhibitor of CDK4/ 6 that prevents $\mathrm{Rb}$ phosphorylation, thus limiting cell-cycle progression [13]. Cell-cycle dysregulation results in uncontrolled cell division, a main indicator of cancer. Targeting specific elements of the cell cycle corrects this discrepancy, contributing to the discovery of potential therapies as well as combination treatment strategies. The cyclin D-CDK4/6-p16-Rb pathway regulates the progression of the cell cycle from the growth phase $\left(G_{1}\right)$ to the DNA synthesis phase $(S)$, and this pathway is frequently altered in breast cancer [4]. In response to mitogenic signaling including estrogen, cyclin D levels increase, resulting in formation of CDK4/6-cyclin D complexes. This subsequently leads to $\mathrm{Rb}$ phosphorylation and release of $\mathrm{E} 2$ transcription factor, which further enables the transcription of genes that lead to cell-cycle progression [5]. Therefore, the cyclin D-CDK4/6-p16-Rb pathway is a potential target for CDK4/6-based cancer therapy.

Currently, three CDK4/6 inhibitors, palbociclib [6], ribociclib [7] and abemaciclib [8], are approved in combination with endocrine therapy (ET) for the treatment of metastatic HR-positive (HR+), HER2-negative (HER2-) breast cancer in the first- or second-line setting on the basis of evidence from Phase III trials where these agents prolonged progression-free survival (PFS) compared with endocrine monotherapy. Current treatment recommendations for postmenopausal women with HR+, HER2- advanced breast cancer (ABC) include various regimens, such as monotherapies (ET, aromatase inhibitors [AIs], fulvestrant) [9] and combinations of targeted therapy and AIs or fulvestrant, based on Phase III trials examining each of the previously mentioned CDK4/6 inhibitors in therapeutic combination with either letrozole, anastrozole or fulvestrant, in addition to the BOLERO-2 trial data evaluating exemestane plus everolimus [10-12].

\section{Introduction to the trial program}

The clinical activity and safety of ribociclib are being investigated across a diverse population of patients through the MONALEESA (Mammary Oncology Assessment of LEE011's Efficacy and Safety) clinical trial program, which includes the MONALEESA-2, MONALEESA-3 and MONALEESA-7 trials. The initial approval of ribociclib was based on results from MONALEESA-2, a Phase III trial that evaluated the combination of ribociclib with letrozole 
as first-line therapy in postmenopausal women with ABC [7]. MONALEESA-3 and MONALEESA-7 are relatively newer trials that are evaluating ribociclib in advanced HR+, HER2- breast cancer populations, with treatment lines and combination partners that differ from those explored in MONALEESA-2 [13-15]. Available results from the MONALEESA program have consistently shown that ribociclib is an effective combination partner with a predictable and manageable safety profile, and the ongoing trials and analyses seek to identify optimal combination regimens in different patient types $[13,14,16]$.

\section{Background \& rationale}

The combination of CDK4/6 inhibitors with AIs as first-line ABC treatment has been studied in other clinical trials, such as PALOMA-2 [17] and MONARCH 3 [18], and owing to the evident therapeutic activity of this combination, it has emerged as a new standard in the first-line treatment setting of postmenopausal women with HR+, HER2$\mathrm{ABC}[11,19]$. In addition, treatment with $\mathrm{CDK} 4 / 6$ inhibitors in combination with fulvestrant has established CDK4/6 inhibitor combination therapy in HR+, HER2- ABC in postmenopausal women who have progressed on antiestrogen therapy [20,21]. Both PALOMA-3 [20] and MONARCH 2 [21] included pre/perimenopausal women; however, these women made up only a minority of the enrolled patients. The MONALEESA trials have further investigated new patient populations and CDK4/6 inhibitor combinations in the first-line ABC treatment setting. MONALEESA-3 is a Phase III study investigating CDK4/6 inhibition plus fulvestrant in de novo HR+, HER2$\mathrm{ABC}$ and as first-line treatment for advanced disease in postmenopausal patients [13]. MONALEESA-7 is the only study to investigate CDK4/6 inhibition in a large population composed entirely of pre/perimenopausal women [14]. On the basis of findings from MONALEESA-3 and MONALEESA-7, ribociclib has been approved for the treatment of $\mathrm{HR}+, \mathrm{HER} 2-\mathrm{ABC}$ in combination with either an $\mathrm{AI}$ or fulvestrant in postmenopausal women and in combination with an $\mathrm{AI}$ in pre/perimenopausal women [22].

\section{Trial design}

\section{Design overview}

The MONALEESA-2, MONALEESA-3 and MONALEESA-7 trials (ClinicalTrials.gov identifiers NCT01958021, NCT02422615 and NCT02278120, respectively) are double-blind, placebo-controlled, randomized Phase III trials. In all three trials, the starting dose of ribociclib was $600 \mathrm{mg}$ once daily for 21 days of a 28-day cycle (3-weeks-on, 1-week-off schedule). In the MONALEESA-2 trial, ribociclib was combined with letrozole (2.5 mg once daily) in postmenopausal women with HR+, HER2- ABC [15]. In the MONALEESA-3 trial, the patient population consisted of postmenopausal women with HR+, HER2- ABC who had relapsed $>12$ months from their adjuvant or neoadjuvant ET or presented with de novo $\mathrm{ABC}$ and were treatment naive or had received $\leq 1$ line of prior ET in the advanced setting. Patients were randomized to receive ribociclib or placebo in combination with fulvestrant $(500 \mathrm{mg}$ intramuscularly on days 1 and 15 of the first 28 -day cycle, then on day 1 of all subsequent 28-day cycles) [13]. In contrast, the MONALEESA-7 trial evaluated pre/perimenopausal patients with HR+, HER2- ABC who received treatment with either ribociclib or placebo with either tamoxifen (20 mg daily) or a nonsteroidal AI (NSAI; letrozole $2.5 \mathrm{mg}$ daily or anastrozole $1 \mathrm{mg}$ daily, both orally) [14]. All patients in MONALEESA-7 received goserelin (3.6 mg subcutaneously on day 1 of every 28-day cycle) for ovarian suppression. In all of the MONALEESA trials, eligibility for study enrollment required the presence of measurable disease according to Response Evaluation Criteria in Solid Tumors (RECIST), version 1.1, or at least one predominantly lytic bone lesion [13-15]. In addition, patients had to have an Eastern Cooperative Oncology Group performance status (ECOG PS) score of 0 or 1 and demonstrate adequate bone marrow and organ function. The presence or absence of liver or lung metastases was a stratification factor in all MONALEESA trials. Adverse events (AEs) were characterized according to Common Terminology Criteria for Adverse Events, version 4.03. Ribociclib dose reductions were standardized across the trials, from 600 to 400 to $200 \mathrm{mg}$ once daily, and were permitted if treatment-related AEs were noted; however, dose reductions were not permitted for letrozole, fulvestrant, tamoxifen, NSAIs or goserelin. Treatment continued until disease progression, unacceptable toxicity, death or discontinuation for any other reason (Figure 1).

\section{Procedures \& outcome measures \\ MONALEESA-2}

Patients were excluded from the study if they received a previous CDK4/6 inhibitor or any previous systemic chemotherapy or ET for advanced disease [15]. Patients with inflammatory breast cancer, CNS metastases, impaired 


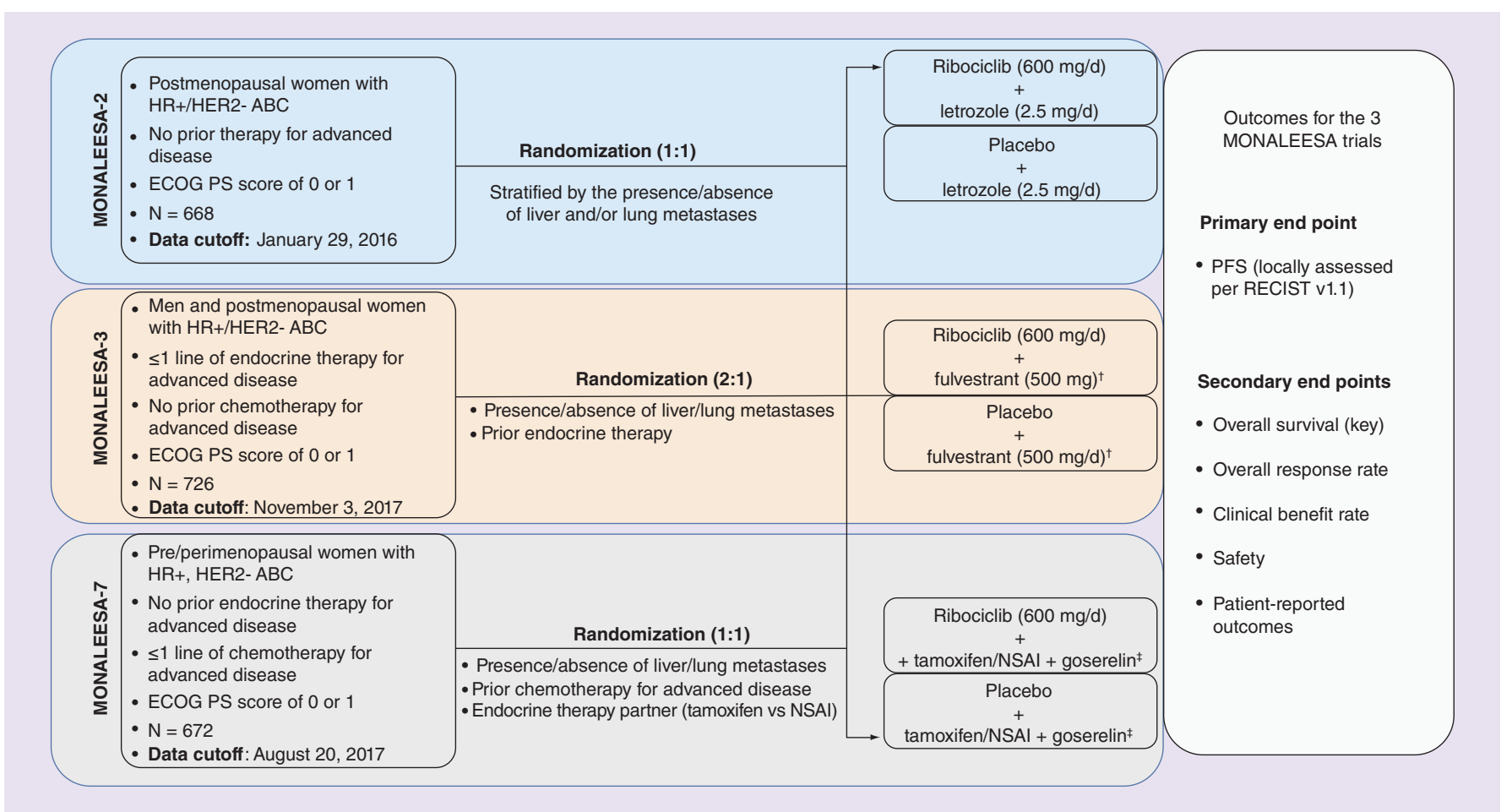

Figure 1. MONALEESA study designs.

${ }^{\dagger}$ Fulvestrant administered on days 1 and 15 of the first 28-day cycle, then on day 1 on all subsequent 28-day cycles. ${ }^{\ddagger}$ Starting dosage for tamoxifen was $20 \mathrm{mg} / \mathrm{d}$, for anastrozole was $1 \mathrm{mg} / \mathrm{d}$, for letrozole was $2.5 \mathrm{mg} / \mathrm{d}$, and for goserelin was $3.6 \mathrm{mg}$ every $28 \mathrm{~d}$.

ECOG PS: Eastern Cooperative Oncology Group performance status; PFS: Progression-free survival; RECIST: Response evaluation criteria in solid tumors.

gastrointestinal function that would alter drug absorption or a history of cardiac disease or dysfunction or baseline corrected QT interval (Fridericia's formula; QTcF) $>450 \mathrm{~ms}$ were also excluded [15]. Previous neoadjuvant or adjuvant use of an NSAI was permitted contingent on a disease-free interval $>12$ months [15]. Patients who discontinued ribociclib or placebo could remain on study, receiving only letrozole, but treatment unblinding and treatment crossover were not permitted [15].

The primary end point was PFS and the secondary end points were overall survival (OS), overall response rate (ORR; complete or partial response), clinical benefit rate (ORR plus stable disease lasting at least 24 weeks), safety, and quality-of-life assessments. Exploratory end points were pharmacokinetics and biomarkers of response or resistance [15]. Tumor assessments were performed during screening, every 8 weeks during the first 18 months, every 12 weeks thereafter until disease progression, and at the end of treatment. All imaging data were reviewed by a blinded independent review committee. ECG evaluations were performed at screening, on day 15 of cycle 1 , and on day 1 of each subsequent cycle until cycle 9. In patients with a mean QTcF $\geq 481 \mathrm{~ms}$ before cycle 10, further ECG measurements were conducted on day 1 of cycle 10 and on day 1 of each subsequent cycle [15]. The recommendation for monitoring neutropenia is a complete blood count $(\mathrm{CBC})$ evaluation before treatment initiation, followed by complete blood count monitoring every 2 weeks for the first two cycles, at the beginning of each of the subsequent 4 cycles, and as clinically indicated [22].

\section{MONALEESA-3}

The MONALEESA-3 trial included a broad range of postmenopausal patients with HR+, HER2- disease, including de novo $\mathrm{ABC}$, regardless of completion of (neo) adjuvant $\mathrm{ET}$, disease-free interval after completion of (neo) adjuvant ET, or receipt of $\leq 1$ prior treatment for $\mathrm{ABC}[13,22]$. Exclusion criteria were having clinically significant cardiac arrhythmias and/or uncontrolled heart disease (including QTcF $>450 \mathrm{~ms}$ ) and having received prior fulvestrant, CDK4/6 inhibitor or chemotherapy for ABC [13]. 
The primary end point was investigator-assessed PFS, with secondary end points including OS, ORR, clinical benefit rate, safety and tolerability [13]. Tumor response was assessed per RECIST, version 1.1, at screening, every 8 weeks after randomization for 18 months and every 12 weeks thereafter until disease progression, death, withdrawal of consent or loss to follow-up [13]. ECG assessments were performed at screening, on day 15 of cycle 1 , on days 1 and 15 of cycle 2 , on day 1 of all subsequent cycles up to cycle 6 , at end of treatment, and as clinically indicated. In patients with a $\mathrm{QTcF} \geq 481 \mathrm{~ms}$ at any time before cycle 7, additional ECGs were performed predose on day 1 of subsequent cycles and post dose every third cycle [13].

\section{MONALEESA-7}

The MONALEESA-7 trial included pre/perimenopausal women aged 18-59 years with HR+, HER2- ABC [14]. Patients with inflammatory breast cancer, CNS metastases, symptomatic visceral disease or clinically significant heart disease or cardiac repolarization abnormality, including QTcF $>450 \mathrm{~ms}$, and patients who received prior CDK4/6 treatment or ET were excluded [14].

The primary end point was PFS and secondary end points included OS, ORR, clinical benefit rate, time to response, duration of response, time to definitive deterioration of ECOG PS, quality of life, and safety and tolerability [14]. Tumor assessments were done using computed tomography or MRI at screening, every 8 weeks during the first 18 months, and every 12 weeks thereafter. Standard ECG assessments were performed at screening, on cycle 1 days 1 and 15, cycle 2 day 1 , cycle 3 day 15, day 1 of all subsequent cycles, and at the end of treatment [14].

\section{Data analysis}

\section{Patient characteristics}

Baseline characteristics and prior treatments of patients who participated in the MONALEESA trials are shown in Table 1. In MONALEESA-2, between January 2014 and March 2015 [15], a total of 668 patients were randomized to receive ribociclib plus letrozole and placebo plus letrozole ( $\mathrm{n}=334$ in each arm), and as of 2 January 2017, 219 patients (32.8\%) remained on study [16]. MONALEESA-3 enrolled 726 postmenopausal women and men with histologically and/or cytologically confirmed HR+, HER2- ABC from June 2015 through June 2016, who were randomly assigned to receive ribociclib plus fulvestrant $(n=484)$ or placebo plus fulvestrant $(\mathrm{n}=242)[13]$. MONALEESA-7 focused on enrollment of only pre/perimenopausal patients and ultimately enrolled and randomized 672 patients between December 2014 and August 2016, with 335 assigned to receive ribociclib plus tamoxifen/NSAI and goserelin and 337 assigned to receive placebo plus tamoxifen/NSAI and goserelin [14]. Despite differences among the study designs of these three trials, the trial characteristics among the ribociclib and placebo arms within the trials were balanced, and the rates were mostly similar. Because patients enrolled in the MONALEESA-7 trial were required to be pre/perimenopausal, the median age was much younger compared with patients in the other two trials [14]. The number of Asian patients enrolled in the MONALEESA-7 trial was also higher than that in the MONALEESA-2 and MONALEESA-3 trials, which was likely due to increased availability of trial sites in Asia in the MONALEESA-7 trial (22 sites in Asian countries vs 14 and nine for MONALEESA-2 and MONALEESA-3, respectively) [23-25].

\section{Efficacy}

In all three trials, the primary end point was met, and PFS was significantly improved in the treatment arm containing ribociclib versus that with the placebo arm. In MONALEESA-2, median PFS in patients who received ribociclib plus letrozole versus placebo plus letrozole was 25.3 versus 16 months (hazard ratio [HR]: 0.568 [95\% CI: $\left.0.457-0.704] ; \mathrm{p}=9.63 \times 10^{-8}\right)$ [16]. In MONALEESA-3, which allowed up to one prior treatment for ABC, the median PFS was 20.5 months (95\% CI: 18.5-23.5 months) versus 12.8 months (95\% CI: 10.9-16.3 months) in the ribociclib plus fulvestrant arm versus the placebo plus fulvestrant arm, and the HR was 0.593 (95\% CI: $0.480-0.732 ; \mathrm{p}<0.001$ ) [13]. Results from the MONALEESA-7 trial also showed a significantly longer duration of PFS in the ribociclib group versus the placebo group [14]. Median PFS was 23.8 months (95\% CI: 19.2 monthsnot reached) in the ribociclib group and 13.0 months (95\% CI: 11.0-16.4 months) in the placebo group (HR: 0.55 [95\% CI: 0.44-0.69]; one-sided $\mathrm{p}<0.001)$. Median PFS in the subgroup of patients receiving tamoxifen as the combination partner $(\mathrm{n}=177)$ was 22.1 months (95\% CI: $16.6-24.7$ months) in the ribociclib group and 11.0 months (95\% CI: 9.1-16.4 months) in the placebo group (HR: 0.59 [95\% CI: 0.39-0.88]). In patients receiving NSAI as the combination partner $(\mathrm{n}=495)$, median PFS was 27.5 months (95\% CI: 19.1 months-not reached) in the ribociclib group and 13.8 months (95\% CI: 12.6-17.4 months) in the placebo group (HR: 0.57 
Table 1. Baseline characteristics.

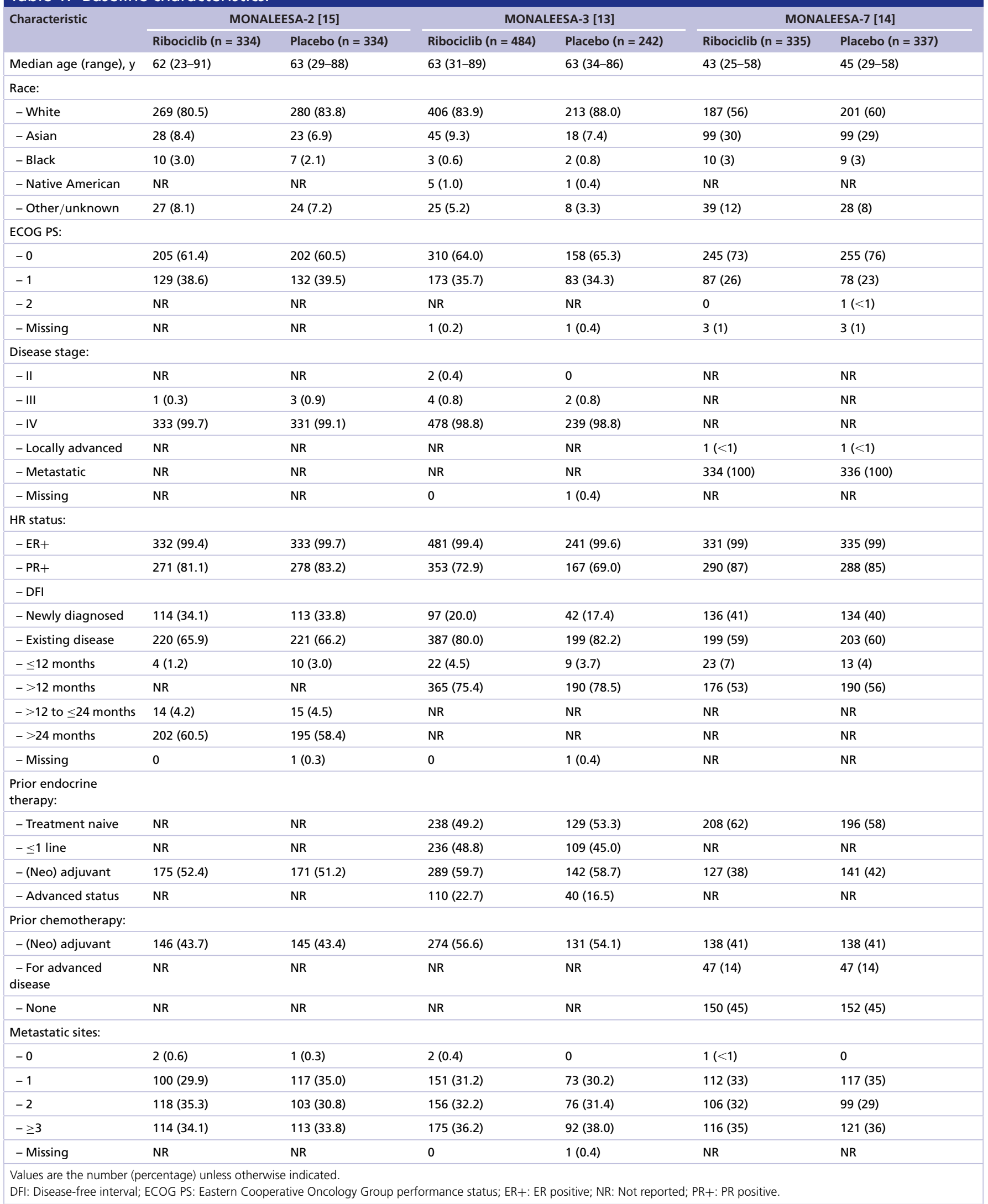


Table 1. Baseline characteristics (cont.).

\begin{tabular}{|c|c|c|c|c|c|c|}
\hline \multirow[t]{2}{*}{ Characteristic } & \multicolumn{2}{|c|}{ MONALEESA-2 [15] } & \multicolumn{2}{|c|}{ MONALEESA-3 [13] } & \multicolumn{2}{|c|}{ MONALEESA-7 [14] } \\
\hline & Ribociclib $(n=334)$ & Placebo $(n=334)$ & Ribociclib $(n=484)$ & Placebo $(n=242)$ & Ribociclib $(n=335)$ & Placebo $(n=337)$ \\
\hline \multicolumn{7}{|l|}{ Site of metastases: } \\
\hline - Bone & $246(73.7)$ & $244(73.1)$ & $367(75.8)$ & $180(74.4)$ & $251(75)$ & $247(73)$ \\
\hline - Bone only & $69(20.7)$ & $78(23.4)$ & $103(21.3)$ & $51(21.1)$ & $81(24)$ & $78(23)$ \\
\hline - Breast & $8(2.4)$ & $11(3.3)$ & $4(0.8)$ & $1(0.4)$ & NR & NR \\
\hline - Visceral & $197(59.0)$ & $196(58.7)$ & $293(60.5)$ & $146(60.3)$ & $193(58)$ & $188(56)$ \\
\hline - Lymph nodes & $133(39.8)$ & $123(36.8)$ & $199(41.1)$ & $115(47.5)$ & $142(42)$ & $158(47)$ \\
\hline - Soft tissue & $N R$ & NR & $23(4.8)$ & $14(5.8)$ & $25(7)$ & $21(6)$ \\
\hline -Skin & NR & NR & $20(4.1)$ & $8(3.3)$ & $8(2)$ & $8(2)$ \\
\hline - Other & $35(10.5)$ & $22(6.6)$ & NR & NR & NR & NR \\
\hline - None & NR & NR & $2(0.4)$ & 0 & NR & NR \\
\hline - Missing & NR & NR & 0 & $1(0.4)$ & NR & NR \\
\hline
\end{tabular}

[95\% CI: 0.44-0.74]). The consistency in the HRs among these three trials underscores the efficacy of CDK4/6 inhibitors across a broad population of patients with HR+, HER2- ABC.

The ORR in the MONALEESA-2 trial was 42.5 versus $28.7 \%(\mathrm{p}<0.0001)$ in the ribociclib group versus the placebo group, and in patients with measurable disease, the ORR was 54.5 versus $38.8 \%(p<0.001)$ [16]. In MONALEESA-3, where about half the patients in the trial were receiving second-line therapy, the ORR was 32.4 versus $21.5 \%(\mathrm{p}<0.001)$ in the ribociclib group versus the placebo group for all patients. In patients with measurable disease, the ORR was 40.9 versus $28.7 \%$ in the ribociclib group versus the placebo group ( $\mathrm{p}$ $=0.003$ [ [13]. The ORR in MONALEESA-7 in all patients was $41 \%$ in the ribociclib group versus $30 \%$ in the placebo group, and among patients with measurable disease, ORR was $51 \%$ and $36 \%$, respectively ( $p<0.001$ for both comparisons) [14]. In all MONALEESA trials, the addition of ribociclib to ET resulted in measurable tumor reductions conferring an advantage in ORR.

On the basis of the available data for the three trials, ribociclib demonstrated benefit in pre-, peri-, and postmenopausal patients in combination with a variety of endocrine agents in the treatment of HR+, HER2$\mathrm{ABC}$ among a diverse population. Subgroup analyses in the MONALEESA trials showed consistent treatment benefit across all subgroups evaluated including age $\geq 65$ years, presence of visceral metastases, bone-only disease, prior (neo)adjuvant chemotherapy or ET and having de novo disease (Table 2). A recent collated subgroup analysis showed that PFS benefit was consistent in patients with visceral metastases regardless of the disease burden $(>3$ visceral metastases); the HR values were 0.60 (95\% CI: 0.40-0.90), 0.52 (95\% CI: 0.26-1.02) and 0.50 (95\% CI: 0.31-0.82) in MONALEESA-2, MONALEESA-3 and MONALEESA-7, respectively [26]. In MONALEESA-2, among patients with non de novo ABC, the PFS benefit of ribociclib plus letrozole was consistent regardless of treatment-free interval (TFI) duration. In patients with TFI $\leq 24$ months, the HR was 0.455 (95\% CI: 0.2960.701 ) and with TFI $>24$ months, the HR was 0.455 (95\% CI: $0.287-0.720$ ) [27]. In patients with TFI $\leq 36$ months and TFI > 36 months, the HR was 0.422 (95\% CI: 0.284-0.627) and 0.507 (95\% CI: 0.303-0.851), respectively. In patients with TFI $\leq 48$ months and TFI $>48$ months, the HR was 0.449 (95\% CI: $0.310-0.650)$ and 0.496 (95\% CI: 0.274-0.898), respectively.

In the MONALEESA-2 and MONALEESA-7 trials, PFS benefit was also observed in Asian patients (Table 2). However, in MONALEESA-3, there was no treatment benefit with ribociclib in Asian patients attributable to the small sample size and limited number of events in this population. Ribociclib plus letrozole has also shown effective tumor reduction versus placebo plus letrozole in MONALEESA-2, where early response was reported in $37.2 \%$ of patients in the ribociclib arm versus $23.3 \%$ of patients in the placebo arm $(n=334$ in each arm) at 6 months [28].

Safety \& tolerability

Overall, the AE profiles of ribociclib in all three trials were similar. The most common AEs of any grade that occurred in $\geq 25 \%$ of patients were neutropenia, leukopenia and nausea (Table 3). In MONALEESA-2, the most common Grade 3/4 AEs ( $\geq 15 \%$ in either arm) were neutropenia and leukopenia [16]. In MONALEESA-3, the 


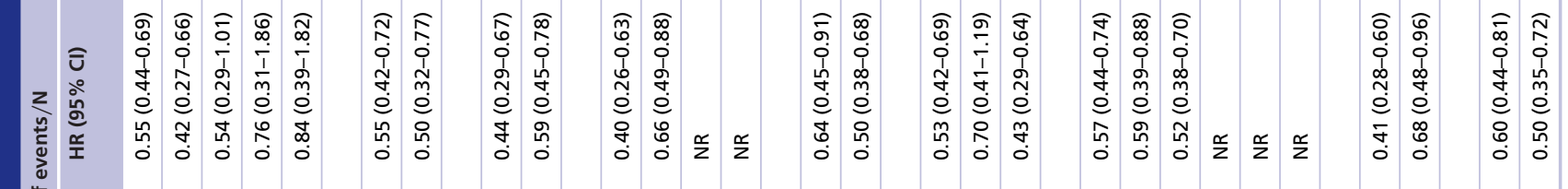
$\stackrel{\circ}{\stackrel{9}{3}}$

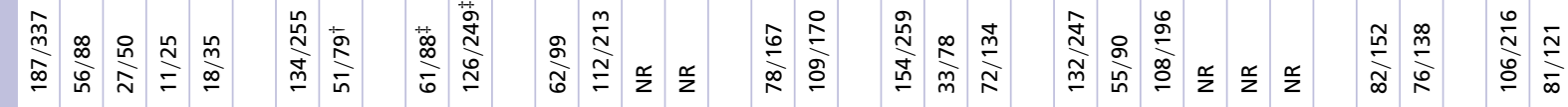
范

$\stackrel{2}{\stackrel{0}{0}}$

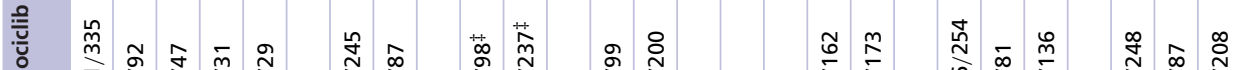

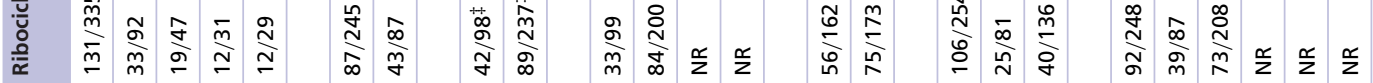

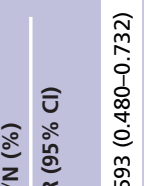

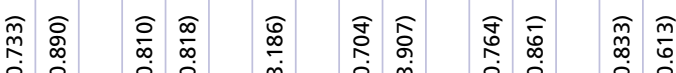

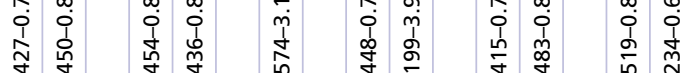

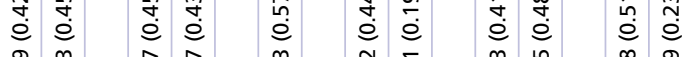

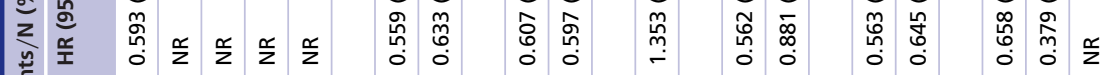

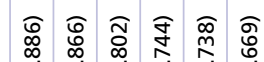

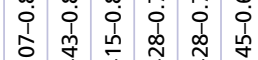

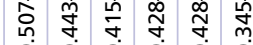

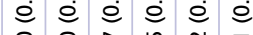

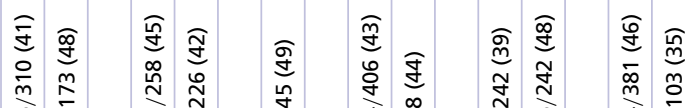

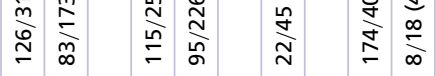

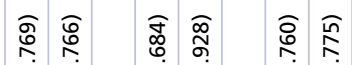

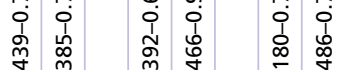

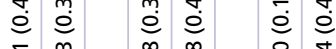

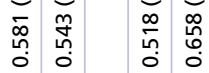

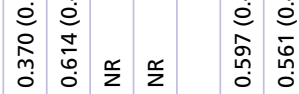

竞

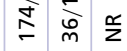

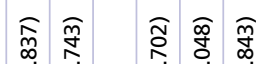

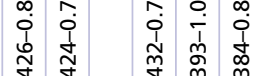

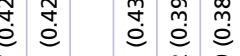

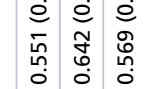

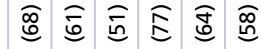

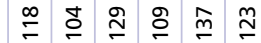

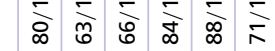

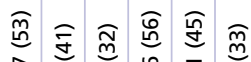

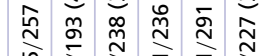

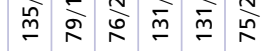
ลิ ๙ิ ๙ิ

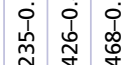

党

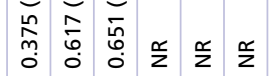

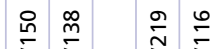

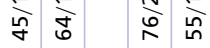

金

辛

อ

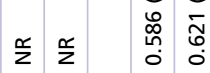

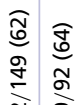

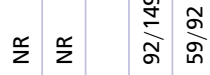

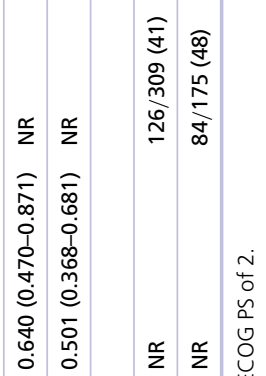

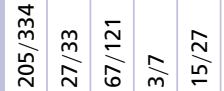

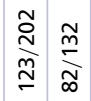

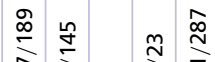

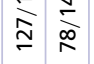

$\stackrel{m}{\frac{\eta}{5}}$

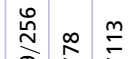

ธิด ํํㅇ

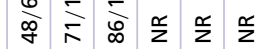

$\stackrel{\infty}{\stackrel{2}{9}}$

종 ํํㅇ $\frac{\infty}{z} \frac{⿱}{z}$

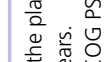

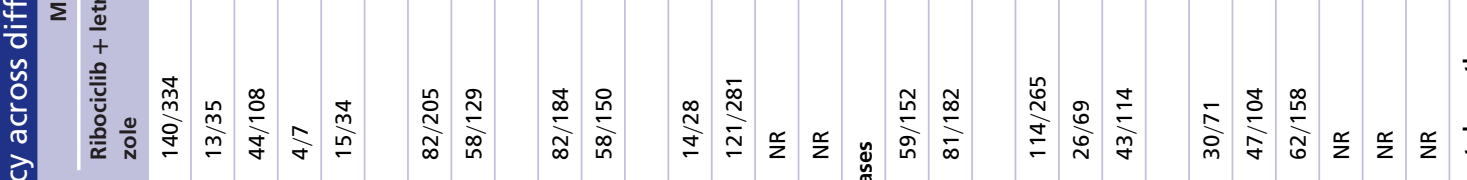




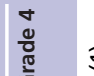

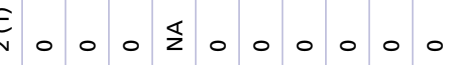

$\stackrel{\overrightarrow{\mathrm{I}}}{\mathrm{\circ}}$

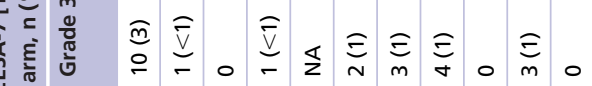

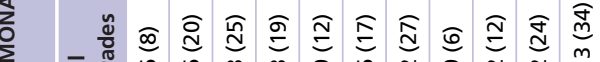

ॠ

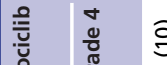

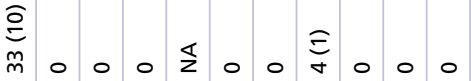

守导 $m$ in

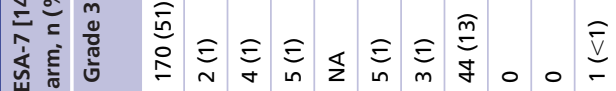

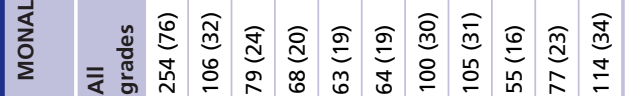

$\therefore$

$\begin{array}{llllllllllllllllllll}\frac{1}{0} & \frac{1}{2} & 0 & 0 & 0 & 0 & 0 & 0 & 0 & 0 & 0 & 0 & 0\end{array}$

mे $m$

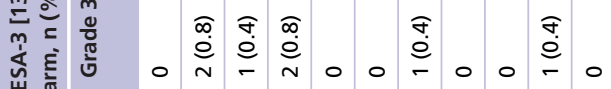

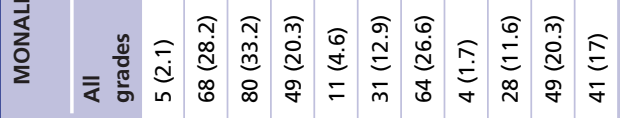

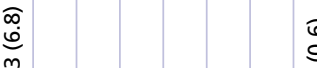

$\stackrel{0}{0}$

m $\mathrm{m} \quad \widehat{6}$

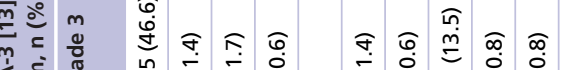

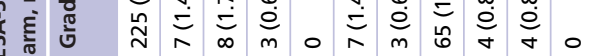

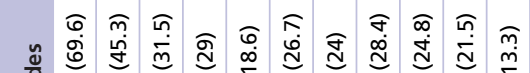

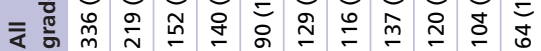

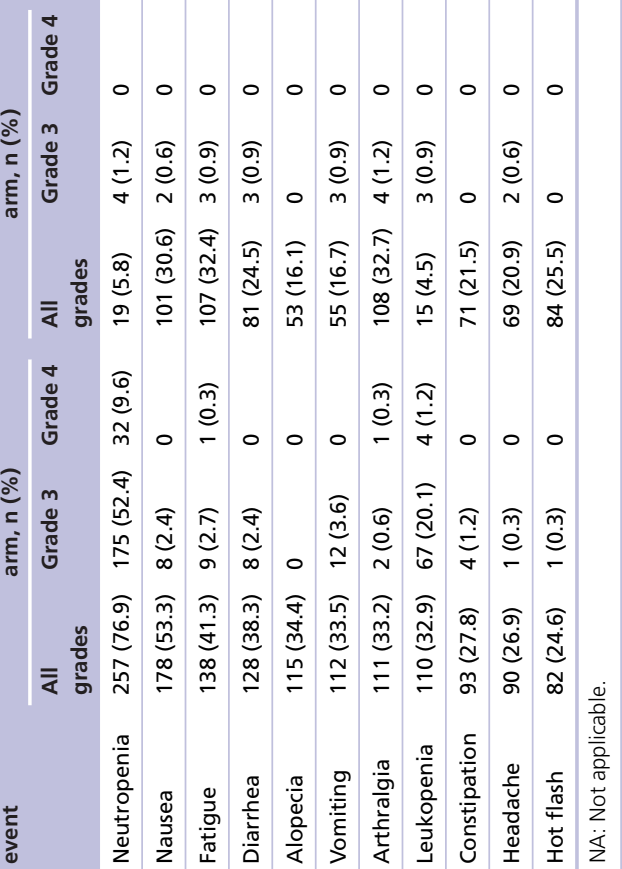


most common Grade 3 AEs occurring in $\geq 10 \%$ of patients were neutropenia and leukopenia [13]. The only Grade $4 \mathrm{AE}$ reported in $\geq 5 \%$ of patients was neutropenia. In MONALEESA-7, the most common Grade $3 / 4 \mathrm{AEs}$ ( $\geq 5 \%$ of the patients in either group) were neutropenia, leukopenia and increased alanine aminotransferase level [14]. Grade $1 / 2$ hot flash was reported at a higher frequency in this trial compared with the others (34\% of patients in each of the ribociclib arm and the placebo arm) possibly because of ovarian function suppression in premenopausal women. Elevated levels of aminotransferases were observed in all three trials, and Grade $3 / 4$ increases were reported in alanine aminotransferase levels ( $10 \mathrm{vs} 2 \%$ ) and aspartate aminotransferase levels ( 7 vs $2 \%)$ in the ribociclib versus placebo arms among all trials [22]. Febrile neutropenia in the ribociclib group was reported in five patients (1.5\%) in the MONALEESA-2 trial, five patients (1\%) in the MONALEESA-3 trial and in seven patients $(2.1 \%)$ in the MONALEESA-7 trial. A collated analysis of the MONALEESA program demonstrated that AEs were effectively managed with dose modifications that primarily occurred within the initial 3 months of treatment, and the efficacy of ribociclib was maintained regardless of dose intensity [29]. Detailed information on dose modifications and management of AEs associated with ribociclib can be found in the package insert [22].

QTcF prolongation was reported in a small number of patients receiving ribociclib in all three trials. In the MONALEESA-2 trial, 10 of 334 patients in the ribociclib group (3\%) experienced a $>60$-ms QTcF prolongation from baseline, and 12 patients (3.6\%) had at least one postbaseline QTcF $>480 \mathrm{~ms}$ versus two of 334 patients $(0.6 \%)$ in the placebo group [16]. In MONALEESA-3, prolongation of QTcF (any grade) was reported in $6.2 \%$ of patients receiving ribociclib plus fulvestrant and $0.8 \%$ of patients receiving placebo plus fulvestrant [13]. Postbaseline QTcF $>480 \mathrm{~ms}$ occurred in $5.6 \%$ of patients in the ribociclib arm and $2.5 \%$ of patients in the placebo arm, and of these patients, 1.7 and $0.4 \%$, respectively, experienced a postbaseline QTcF $>500 \mathrm{~ms}$. An increase of $>60 \mathrm{~ms}$ from baseline in QTcF occurred in 6.5 and $0.4 \%$ of patients randomized to ribociclib plus fulvestrant and placebo plus fulvestrant, respectively. Three patients $(0.6 \%)$ in the ribociclib plus fulvestrant arm and no patients in the placebo plus fulvestrant arm discontinued study treatment because of QTcF prolongation. In MONALEESA-7, a postbaseline QTcF > $480 \mathrm{~ms}$ occurred in 23 patients (6.9\%) in the ribociclib group and in four patients (1.2\%) in the placebo group, and among these patients, postbaseline QTcF $>500 \mathrm{~ms}$ was reported in five patients (1.5\%) and one patient (0.3\%), respectively [14]. A $>60$-ms increase in QTcF from baseline occurred in 32 patients (9.6\%) in the ribociclib group and in six patients $(1.8 \%)$ in the placebo group. In the ribociclib group, a $>60-\mathrm{ms}$ increase from baseline in QTcF was observed in 14 of 87 patients (16\%) receiving tamoxifen and in 18 of 245 patients (7\%) receiving NSAI [22]. In the placebo group, an increase of $>60 \mathrm{~ms}$ from baseline occurred in six of 90 patients (7\%) receiving tamoxifen and in no patients receiving NSAI. However, none of the patients had clinical symptoms or arrhythmias. During treatment with ribociclib, more frequent ECG monitoring is recommended in case of QTcF prolongation at any given time [22].

Of note, ribociclib is not indicated in combination with tamoxifen for the treatment of premenopausal women with ABC. Ribociclib use should also be avoided with strong cytochrome P4503A inhibitors and other drugs that prolong the QT interval [22]. Detailed information on drug-drug interactions with ribociclib can be found in the Package Insert [22].

\section{Health-related quality of life}

Overall health-related quality of life was maintained from baseline in both arms of the MONALEESA-2 trial in subgroups of patients with visceral disease, bone-only metastases, best overall response of complete or partial response and ECOG PS score of 0 or 1 [30]. Clinically meaningful reduction ( $>5$ points) in the European Organisation for Research and Treatment of Cancer Quality of Life Core 30 Questionnaire (EORTC QLQ-C30) pain score was observed at week 8 and was maintained up to cycle 11 in the ribociclib arm [30,31]. A greater improvement in pain from baseline was reported in the ribociclib arm (26\%) versus that in the placebo arm (15\%) [28]. The percent change from baseline in EORTC QLQ-C30 pain score in patients receiving ribociclib was -40 versus $-29 \%$ in those receiving placebo, and in patients with clinical benefit, the mean change in pain score from baseline was -7 and -1.3 points in the ribociclib and placebo arms, respectively [28]. In the MONALEESA-3 and MONALEESA-7 trials, time to definitive deterioration was $\geq 10 \%$ in the ribociclib arm versus placebo arm [32-34]. Global health-related quality of life was improved or maintained during treatment compared with baseline and worsened upon treatment discontinuation in both arms in both trials. In MONALEESA-7, a reduction in pain was observed at week 8 and maintained up to cycle 19 in the ribociclib arm [33]. 


\section{Conclusion}

The overall findings from the MONALEESA program highlight the treatment benefit and manageable safety profile of ribociclib in combination with AIs or fulvestrant compared with placebo, and in different patient subpopulations with HR+, HER2- ABC. Most of the AEs reported across these trials were consistent. Careful monitoring and dose modifications are recommended and should be implemented as needed in patients with a high risk of QTcF prolongation.

The MONALEESA clinical program for ribociclib includes diverse patient populations in different treatment settings. Besides the available clinical evidence that supports the widespread use of ribociclib, ongoing clinical investigations are further exploring other applications and characteristics of ribociclib with different combination agents, in different patient populations and in other treatment settings. For instance, to further characterize the efficacy and safety of ribociclib in a large and diverse patient population with HR+, HER2- ABC, a Phase IIIb openlabel, single-arm, multicenter trial, the CompLEEment-1 trial (ClinicalTrials.gov identifier: NCT02941926), is currently ongoing (enrollment is 3256 patients). Preliminary results from the initial 1008 patients with any menopausal status enrolled with 56 days of follow-up demonstrated a safety profile of ribociclib plus letrozole consistent with that of the MONALEESA-2 trial of postmenopausal women receiving first-line treatment for HR+, HER2- ABC [35]. Another ongoing trial, the TRINITI-1 trial (ClinicalTrials.gov identifier: NCT02732119), is investigating the effects of continuing a CDK4/6 inhibitor through triplet therapy (ribociclib plus everolimus plus exemestane) in patients with disease progression on a CDK4/6 inhibitor [36. Furthermore, the disposition of ribociclib in the CNS is also being investigated in preclinical and early clinical studies. Preclinical results in a mouse model have shown adequate CNS penetration, which resulted in subsequent preclinical efficacy studies [37]. Results from a Phase I, multicenter, open-label, dose-escalation study of ribociclib (ClinicalTrials.gov identifier: NCT01747876) in 32 pediatric patients with malignant rhabdoid tumor or neuroblastoma also showed rapid absorption of ribociclib, despite some interpatient variability and a predictable safety profile [38].

Overall survival data with ribociclib for the treatment of HR+, HER2- ABC in a Phase III setting are not yet available and are currently being investigated. Mixed results in OS data with the CDK4/6 inhibitor palbociclib have been observed in the PALOMA-1 and PALOMA-3 trials [39,40]. PALOMA-3 demonstrated improved OS in patients with sensitivity to previous ET treated with palbociclib plus fulvestrant compared with that in patients treated with placebo plus fulvestrant (39.7 vs 29.7 mo, respectively; HR for death, 0.72 [95\% CI: 0.55-0.93]) [40]; however, there was not a significant OS benefit in the entire patient population. No significant OS benefit was observed with treatment with palbociclib plus letrozole versus placebo plus letrozole in the Phase II PALOMA-1 study [39]. Furthermore, interpretation of OS data in the PALOMA trials is confounded by postprogression therapies after study discontinuation. For example, the length of OS relative to PFS in PALOMA-3 indicates that many patients will be treated with at least one postprogression therapy for years following study treatment discontinuation.

Ribociclib is now being investigated as a potential adjuvant or neoadjuvant therapy in patients with breast cancer. These trials are randomizing HR+, HER2- breast cancer patients to treatment with ribociclib in combination with ET or to standard of care treatment. In the neoadjuvant setting, the Phase II FELINE trial is comparing ribociclib plus letrozole versus placebo plus letrozole in postmenopausal women with HR+, HER2early breast cancer (NCTT02712723) [41,42]; the Phase II NEOLBC trial is comparing ribociclib plus letrozole versus standard chemotherapy as a neoadjuvant therapy in postmenopausal women with HR+, HER2- luminal breast cancer (NCT03283384) [43]; and the CORALLEEN trial is comparing ribociclib plus letrozole versus standard chemotherapy as a neoadjuvant therapy in postmenopausal women with luminal B, HER2- breast cancer (NCT03248427) [44,45]. In addition, the Phase III NATALEE trial will evaluate the efficacy and safety of ribociclib plus ET compared with ET alone as an adjuvant treatment in pre/perimenopausal and postmenopausal women with early breast cancer (NCT03701334) [46].

Despite ET being the primary treatment option, the combinations of ribociclib with AIs or fulvestrant are becoming preferred ET combinations in patients with HR+, HER2- ABC in the first- or second-line disease setting. The addition of ribociclib with other established therapeutic agents has become an important option to improve PFS and overcome ET resistance. 
- The clinical activity and safety of ribociclib, an orally bioavailable, selective inhibitor of CDK4/6, are being investigated in the MONALEESA clinical trial program that includes the MONALEESA-2, MONALEESA-3 and MONALEESA-7 trials.

Design

- In all three double-blind, placebo-controlled, randomized Phase III trials in patients with HR-positive, HER2-negative advanced breast cancer, the starting dose of ribociclib was $600 \mathrm{mg}$ once daily for 21 days of a 28-day cycle (3-weeks-on, 1-week-off schedule).

- Postmenopausal women received ribociclib plus letrozole ( $2.5 \mathrm{mg}$ once daily) in the MONALEESA-2 trial.

- The MONALEESA-3 trial evaluated ribociclib plus fulvestrant (500 mg intramuscularly) in postmenopausal women who had relapsed $>12$ months from their endocrine therapy or presented with de novo advanced breast cancer and were treatment naive or had received $\leq 1$ line of prior endocrine therapy in the advanced setting.

- In the MONALEESA-7 trial, pre/perimenopausal women received ribociclib plus goserelin (3.6 mg) with either tamoxifen ( $20 \mathrm{mg}$ daily) or a nonsteroidal aromatase inhibitor (letrozole $2.5 \mathrm{mg}$ daily or anastrozole $1 \mathrm{mg}$ daily).

Data analysis

Efficacy

- In all three trials, the primary end point was met, and progression-free survival (PFS) was significantly improved in the treatment arm containing ribociclib versus that in the placebo arm.

- In MONALEESA-2, the median PFS in patients who received ribociclib was 25.3 versus 16 months in those who received placebo (hazard ratio [HR]: 0.568 [95\% Cl: $0.457-0.704] ; \mathrm{p}=9.63 \times 10^{-8}$ ).

- In MONALEESA-3, the median PFS was 20.5 months (95\% Cl: $18.5-23.5$ months) and 12.8 months (95\% Cl: 10.9-16.3 months) in the ribociclib and placebo arm, respectively, and the HR was 0.593 (95\% Cl: $0.480-0.732 ; \mathrm{p}$ $<0.001)$.

- In MONALEESA-7, median PFS was 23.8 months ( $95 \% \mathrm{Cl}: 19.2$ months-not reached) in the ribociclib group and 13.0 months ( $95 \%$ Cl: 11.0-16.4 months) in the placebo group (HR: 0.55 [95\% Cl: 0.44-0.69]; one-sided p < 0.001).

- Median PFS in the subgroup of patients receiving tamoxifen as the combination partner $(n=177)$ was 22.1 months ( $95 \% \mathrm{Cl}: 16.6-24.7$ months) in the ribociclib group and 11.0 months ( $95 \% \mathrm{Cl}: 9.1-16.4$ months) in the placebo group (HR: 0.59 [95\% Cl: 0.39-0.88]).

- In patients receiving a nonsteroidal aromatase inhibitor as the combination partner $(n=495)$, median PFS was 27.5 months $(95 \% \mathrm{Cl}: 19.1$ months-not reached) in the ribociclib group and 13.8 months (95\% Cl: 12.6-17.4 months) in the placebo group (HR: 0.57 [95\% Cl: 0.44-0.74]).

Safety

- Overall, the adverse event profiles of ribociclib in all three trials were similar.

- The most common adverse events of any grade that occurred in $\geq 25 \%$ of patients were neutropenia, leukopenia and nausea.

- Corrected QT interval (Fridericia's formula) prolongation was reported in $<10 \%$ of patients receiving ribociclib in each of the three trials.

\section{Author's contributions}

DA Yardley contributed to the design of the manuscript, critically revised the manuscript, and read and approved all drafts.

Financial \& competing interests disclosure

This study was funded by Novartis Pharmaceuticals Corporation. DA Yardley has received grant support outside of the supported work from AbbVie, Amgen, Astellas, AstraZeneca, Bayer, BioMarin, Biothera, Bristol-Myers Squibb, Celgene, Celldex, Clovis, Concordia, Eli Lilly, Eisai, Exelixis, G1 Therapeutics, Genzyme, GlaxoSmithKline, ImClone, Incyte, Ipsen, Janssen, Medimmune, Medivation, Merck, Merrimack, Novartis, Pfizer, Roche/Genentech, Sanofi, Spectrum, and Tesaro and has received consulting fees outside of the supported work from Abraxis, Biotheranostics, Celgene, Daiichi Sankyo, Eisai, Eli Lilly, ELM Medical Limited, Merck, NipponKayaku, Novartis, Pfizer, R-Pharm US, Roche/Genentech and Spectrum. The author has no other relevant affiliations or financial involvement with any organization or entity with a financial interest in or financial conflict with the subject matter or materials discussed in the manuscript apart from those disclosed.

Medical writing and editorial assistance was provided under the direction of the author by R Parthasarathy and D Boffa, ELS, MedThink SciCom (Cary, NC) and was funded by Novartis Pharmaceuticals Corporation.

Ethical conduct of research

This review article does not contain any studies with human participants or animals performed by the author. 
Open access

This work is licensed under the Attribution-NonCommercial-NoDerivatives 4.0 Unported License. To view a copy of this license, visit http://creativecommons.org/licenses/by-nc-nd/4.0/

\section{References}

Papers of special note have been highlighted as: $\bullet$ of interest; $\bullet \bullet$ of considerable interest

1. Kim S, Tiedt R, Loo A et al. The potent and selective cyclin-dependent kinases 4 and 6 inhibitor ribociclib (LEE011) is a versatile combination partner in preclinical cancer models. Oncotarget 9(81), 35226-35240 (2018).

- Provides a detailed report of the preclinical profile of ribociclib in both single-agent and combination settings.

2. Kim S, Loo A, Chopra R et al. Lee011: an orally bioavailable, selective small molecule inhibitor of CDK4/6 - reactivating Rb in cancer. Presented at: AACR-NCI-EORTC International Conference: Molecular Targets and Cancer Therapeutics. Boston, MA, USA, 19-23 October 2013.

3. Rader J, Russell MR, Hart LS et al. Dual CDK4/CDK6 inhibition induces cell-cycle arrest and senescence in neuroblastoma. Clin. Cancer. Res. 19(22), 6173-6182 (2013).

4. Caldon CE, Daly RJ, Sutherland RL, Musgrove EA. Cell cycle control in breast cancer cells. J. Cell. Biochem. 97(2), 261-274 (2006).

5. Abukhdeir AM, Park BH. P21 and p27: roles in carcinogenesis and drug resistance. Expert. Rev. Mol. Med. 10, e19 (2008).

6. Walker AJ, Wedam S, Amiri-Kordestani L et al. FDA approval of palbociclib in combination with fulvestrant for the treatment of hormone receptor-positive, HER2-negative metastatic breast cancer. Clin. Cancer. Res. 22(20), 4968-4972 (2016).

7. Shah A, Bloomquist E, Tang $S$ et al. FDA approval: ribociclib for the treatment of postmenopausal women with hormone receptor-positive, HER2-negative advanced or metastatic breast cancer. Clin. Cancer. Res. 24(13), 2999-3004 (2018).

8. Kim ES. Abemaciclib: first global approval. Drugs 77(18), 2063-2070 (2017).

9. Tong CWS, Wu M, Cho WCS, To KKW. Recent advances in the treatment of breast cancer. Front Oncol. 8, 227 (2018).

10. Ayyagari R, Tang D, Patterson-Lomba $\mathrm{O}$ et al. Progression-free survival with endocrine-based therapies following progression on non-steroidal aromatase inhibitor among postmenopausal women with hormone receptor positive, human epidermal growth factor receptor-2 negative metastatic breast cancer: a network meta-analysis. Curr. Med. Res. Opin. 34(9), 1645-1652 (2018).

11. Referenced with permission from the NCCN Clinical Practice Guidelines in Breast Cancer V.4.2018. ( $)$ National Comprehensive Cancer Network, Inc. 2019. All rights reserved. Accesssed March 6, 2019. To view the most recent and complete verison of the guideline, go online to NCCN.org. NCCN makes no warranties of any kind whatsoever regarding their content, use or application and disclaims any responsibility for their application or use in any way.

12. Baselga J, Campone M, Piccart $\mathrm{M}$ et al. Everolimus in postmenopausal hormone-receptor-positive advanced breast cancer. $N$. Engl. J. Med. 366(6), 520-529 (2012).

13. Slamon DJ, Neven P, Chia $S$ et al. Phase III randomized study of ribociclib and fulvestrant in hormone receptor-positive, human epidermal growth factor receptor 2-negative advanced breast cancer: MONALEESA-3. J. Clin. Oncol. 36(24), 2465-2472 (2018).

-• Discusses findings from a Phase III study that evaluated ribociclib plus fulvestrant in patients with hormone receptor-positive, human epidermal growth factor receptor 2 -negative advanced breast cancer who were treatment-naive in the advanced setting or had received $\leq 1$ line of prior endocrine therapy for advanced disease.

14. Tripathy D, Im SA, Colleoni M et al. Ribociclib plus endocrine therapy for premenopausal women with hormone-receptor-positive, advanced breast cancer (MONALEESA-7): a randomised Phase 3 trial. Lancet. Oncol. 19(7), 904-915 (2018).

-. Provides evidence that ribociclib in combination with endocrine therapy plus ovarian suppression with goserelin is effective and well tolerated in premenopausal women with hormone receptor-positive, human epidermal growth factor receptor 2-negative advanced breast cancer.

15. Hortobagyi GN, Stemmer SM, Burris HA et al. Ribociclib as first-line therapy for HR-positive, advanced breast cancer. N. Engl. J. Med. 375(18), 1738-1748 (2016).

-. Reports efficacy and safety data of the combination of ribociclib and letrozole as initial therapy in patients with hormone receptor-positive, human epidermal growth factor receptor 2-negative advanced breast cancer from MONALEESA-2, a Phase III trial. The approval of ribociclib for treatment was based on data from this trial.

16. Hortobagyi GN, Stemmer SM, Burris HA et al. Updated results from MONALEESA-2, a Phase III trial of first-line ribociclib plus letrozole versus placebo plus letrozole in hormone receptor-positive, HER2-negative advanced breast cancer. Ann. Oncol. 29(7), 1541-1547 (2018).

- Describes updated efficacy and safety data from the MONALEESA-2 trial with a longer follow-up. Exploratory biomarker analyses are also reported.

17. Finn RS, Crown JP, Ettl J et al. Efficacy and safety of palbociclib in combination with letrozole as first-line treatment of ER-positive, HER2-negative, advanced breast cancer: expanded analyses of subgroups from the randomized pivotal trial PALOMA-1/TRIO-18. Breast Cancer Res. 18(1), 67 (2016). 
18. Goetz MP, Toi M, Campone M et al. MONARCH 3: abemaciclib as initial therapy for advanced breast cancer. J. Clin. Oncol. 35(32), 3638-3646 (2017).

19. Pernas S, Tolaney SM, Winer EP, Goel S. CDK4/6 inhibition in breast cancer: current practice and future directions. Ther. Adv. Med. Oncol. 10, 1758835918786451 (2018).

- Provides a comprehensive review of the use of cyclin-dependent kinases 4 and 6 inhibitors in breast cancer and discusses their use in clinical practice.

20. Cristofanilli M, Turner NC, Bondarenko I et al. Fulvestrant plus palbociclib versus fulvestrant plus placebo for treatment of hormone-receptor-positive, HER2-negative metastatic breast cancer that progressed on previous endocrine therapy (PALOMA-3): final analysis of the multicentre, double-blind, Phase 3 randomised controlled trial. Lancet Oncol. 17(4), 425-439 (2016).

21. Sledge GW Jr, Toi M, Neven P et al. MONARCH 2: abemaciclib in combination with fulvestrant in women with HR+/HER2advanced breast cancer who had progressed while receiving endocrine therapy. J. Clin. Oncol. 35(25), 2875-2884 (2017).

22. Kisqali [package insert]. Novartis Pharmaceuticals Corp., NJ, USA (2018).

23. ClinicalTrials.gov. Study of Efficacy and Safety of LEE011 in Postmenopausal Women With Advanced Breast Cancer (MONALEESA-2). https://clinicaltrials.gov/ct2/show/NCT01958021

24. ClinicalTrials.gov. Study of Efficacy and Safety of LEE011 in Men and Postmenopausal Women With Advanced Breast Cancer (MONALEESA-3). https://clinicaltrials.gov/ct2/show/NCT02422615

25. ClinicalTrials.gov. Study of Efficacy and Safety in Premenopausal Women With Hormone Receptor Positive, HER2-negative Advanced Breast Cancer (MONALEESA-7). https://clinicaltrials.gov/ct2/show/NCT02278120

26. Yardley D, Chan A, Nusch A et al. Ribociclib plus endocrine therapy in patients with hormone receptor-positive, HER2-negative advanced breast cancer presenting with visceral metastases: subgroup analysis of Phase 3 MONALEESA trials. Presented at: San Antonio Breast Cancer Symposium. San Antonio, TX, USA, 4-8 December 2018.

27. Blackwell KL, Paluch-Shimon S, Campone M et al. Subsequent treatment for postmenopausal women with hormone receptor-positive, HER2-negative advanced breast cancer who received ribociclib + letrozole vs placebo + letrozole in the Phase III MONALEESA-2 study. Presented at: San Antonio Breast Cancer Symposium. TX, USA, 5-9 December 2017.

28. Janni W, Alba E, Bachelot T et al. First-line ribociclib plus letrozole in postmenopausal women with HR+, HER2-advanced breast cancer: tumor response and pain reduction in the Phase 3 MONALEESA-2 trial. Breast. Cancer. Res. Treat. 169(3), 469-479 (2018).

29. Beck JT, Neven P, Sohn J et al. Ribociclib treatment benefit in patients with advanced breast cancer with $\geq 1$ dose reduction: data from the MONALEESA-2, -3, and -7 trials. Presented at: San Antonio Breast Cancer Symposium. San Antonio, TX, USA, 4-8 December 2018.

30. Verma S, O'Shaughnessy J, Burris HA et al. Health-related quality of life of postmenopausal women with hormone receptor-positive, human epidermal growth factor receptor 2-negative advanced breast cancer treated with ribociclib + letrozole: results from MONALEESA-2. Breast Cancer Res. Treat. 170(3), 535-545 (2018).

31. Burris HA, Tolaney SM, Hart LL et al. Maintenance of health-related quality of life in elderly patients treated with ribociclib + letrozole in MONALEESA-2. Presented at: American Society of Clinical Oncology Annual Meeting. Chicago, IL, USA, 1-5 June 2018.

32. Harbeck N, Villanueva Vazquez R, Franke F et al. Ribociclib + tamoxifen or a non-steroidal aromatase inhibitor in premenopausal patients with hormone receptor-positive, HER2-negative advanced breast cancer: MONALEESA-7 patient-reported outcomes. Presented at: European Society for Medical Oncology Congress 2018. Munich, Germany, 19-23 October 2018.

33. Harbeck N, Villanueva Vazquez R, Tripathy D et al. Ribociclib (RIB) plus tamoxifen (TAM) or a non-steroidal aromatase inhibitor (NSAI) in premenopausal women with hormone receptorpositive (HR+), human epidermal growth factor receptor 2-negative (HER2-) advanced breast cancer (ABC): additional results from the MONALEESA-7 trial. Eur. J. Cancer 92(Suppl 3), S1 (2018).

34. Fasching PA, Esteva FJ, Pivot X et al. Patient-reported outcomes (PROs) in advanced breast cancer (ABC) treated with ribociclib + fulvestrant: results from MONALEESA-3. Presented at: European Society for Medical Oncology Congress 2018. Munich, Germany, 19-23 October 2018.

35. De Laurentiis $M$, Neven $P$, Jerusalem G et al. Ribociclib + letrozole in patients with hormone receptor-positive, human epidermal growth factor receptor 2-negative advanced breast cancer $(\mathrm{ABC})$ with no prior endocrine therapy for $\mathrm{ABC}$ : preliminary results from the Phase 3b CompLEEment-1 trial. Presented at: American Society of Clinical Oncology Annual Meeting. Chicago, IL, USA, 1-5 June 2018.

36. Moulder S, Karuturi M, Yardley DA et al. Ribociclib in combination with everolimus and exemestane in men and postmenopausal women with HR+/HER2 - advanced breast cancer after progression on a CDK4/6 inhibitor: efficacy and safety results from Phase 2 of the TRINITI-1 study. Presented at: American Association for Cancer Research Annual Meeting. Chicago, IL, USA, 14-18 April 2018.

37. Patel Y, Davis A, Kala A et al. CNS penetration of the CD4/6 inhibitor ribociclib (LEE011) in non-tumor bearing mice and mice bearing orthotopic pediatric brain tumors. Neuro Oncol. 18, vi152 (2018).

38. Geoerger B, Bourdeaut F, Dubois SG et al. A Phase I study of the CDK4/6 inhibitor ribociclib (LEE011) in pediatric patients with malignant rhabdoid tumors, neuroblastoma, and other solid tumors. Clin. Cancer Res. 23(10), 2433-2441 (2017).

39. Finn R, Crown J, Lang I et al. Overall survival results from the randomized Phase II study of palbociclib (P) in combination with letrozole (L) vs letrozole alone for frontline treatment of ER+/HER2- advanced breast cancer (PALOMA-1; TRIO-18). Presented at: American Society of Clinical Oncology Annual Meeting. Chicago, IL, USA, 2-6 June 2017. 
40. Turner NC, Slamon DJ, Ro J et al. Overall survival with palbociclib and fulvestrant in advanced breast cancer. N. Engl. J. Med. 379(20), 1926-1936 (2018).

41. Khan Q, Prochaska L, Mohammad J et al. Femara plus ribociclib or placebo as neo-adjuvant endocrine therapy for women with ER+, HER2-negative early breast cancer - The Feline trial. Presented at: 2016 San Antonio Breast Cancer Symposium. San Antonio, TX, USA, 6-10 December 2016.

42. ClinicalTrials.gov. Letrozole plus ribociclib or placebo as neo-adjuvant therapy in ER-positive HER2-negative early breast cancer (FELINE). https://clinicaltrials.gov/ct2/show/NCT02712723

43. ClincalTrials.gov. Tailoring NEOadjuvant therapy in hormone receptor positive, HER2 negative, luminal breast cancer. (NEOLBC). https://clinicaltrials.gov/ct2/show/NCT03283384

44. Gavilá J, Saura C, Oliveira M et al. CORALLEEN: a Phase 2 clinical trial of chemotherapy or letrozole plus ribociclib as neoadjuvant treatment for postmenopausal patients with luminal B/HER2-negative breast cancer. J. Clin. Oncol. 35 (15 Suppl.), TPS594-TPS594 (2017).

45. ClinicalTrials.gov. Neadjuvant multi-agent chemotherapy or letrozole plus ribociclib in luminal B/Her2-negative breast cancer. (Coralleen). https://clinicaltrials.gov/ct2/show/nct03248427

46. ClinicalTrials.gov. A trial to evaluate efficacy and safety of ribociclib with endocrine therapy as adjuvant treatment in patients with HR+/HER2- early breast cancer (NATALEE). https://clinicaltrials.gov/ct2/show/NCT03701334 